\title{
Adaptive Physical Layer Selection for Bluetooth 5: Measurements and Simulations
}

\author{
Muhammad Usman Sheikh $\left(\mathbb{D}\right.$, Behnam Badihi $\mathbb{D}^{D}$, Kalle Ruttik $(\mathbb{D})$, and Riku Jäntti \\ Department of Communications and Networking, Aalto University, 02150 Espoo, Finland \\ Correspondence should be addressed to Muhammad Usman Sheikh; muhammad.sheikh@aalto.fi
}

Received 8 July 2020; Revised 4 December 2020; Accepted 16 December 2020; Published 6 January 2021

Academic Editor: Carlo Giannelli

Copyright (c) 2021 Muhammad Usman Sheikh et al. This is an open access article distributed under the Creative Commons Attribution License, which permits unrestricted use, distribution, and reproduction in any medium, provided the original work is properly cited.

\begin{abstract}
The main target of this article is to propose a simple Received Signal Strength Indicator- (RSSI-) based adaptive Physical Layer (PHY) selection approach for Bluetooth Low Energy 5.0 (BLE 5) and compare the performance of adaptive PHY selection with the performance of individual PHY. This article also validates the simulation results acquired from the in-house developed Ray Tracing (RT) tool with the BLE 5 measurements conducted in a corridor of the TUAS building, Aalto University. The performance metrics considered for the analysis are RSSI, throughput, and outage ratio. The impact of different transmission power on BLE 5 is also analyzed. The obtained simulation results indicate a Root Mean Square Error (RMSE) of about $4.6 \mathrm{~dB}$ between the measured and the simulated RSSI at $2.4 \mathrm{GHz}$ frequency. By investigating these results, an adaptive PHY selection algorithm is proposed in this article. The adaptive PHY selection approach enhances the mean throughput of the system and improves the outage ratio as well. In the light of acquired results, the adaptive PHY selection scheme shows significant performance gain and is found more beneficial in the case of an environment with mixed RSSI samples, i.e., a mix of low and high RSSI values.
\end{abstract}

\section{Introduction}

In recent decades, the explosive growth of the Internet and advancement in emerging pervasive and miniaturized computing devices have deeply modified the quality of life [1]. The technological paradigm shift called as Internet of Things (IoT) has changed the concept of connectivity from only humans to everything capable of being connected [2]. Several communication technologies are required to make IoT fully functional [3]. Among those, Bluetooth Low Energy (BLE) is considered as an important short-range radio technology for enabling IoT use-cases in Personal Area Networks (PANs) and Body Area Networks (BANs).

In the recent past, BLE also known as Bluetooth smart has gained much success and popularity due to the applicability of BLE in various application areas [4]. BLE is considered as an energy-efficient low-power, low-cost, and less complex short-range radio technology designed for light duty-cycled data exchange. BLE was first introduced by Nokia in 2006, and then, it was added to the Bluetooth
Version 4.0 Core Specification in 2010 [5]. Ever since, several versions have been standardized and improved the overall performance of BLE. Lately, Bluetooth Special Interest Group (SIG) [6] released BLE version 5.0 in Dec. 2016 and version 5.1 in Jan. 2019 to meet the versatile requirements of the upcoming wave of IoT applications and use-cases [7].

BLE 5 is equipped with new features, e.g., increased communication range, higher data rate, greater broadcast capacity, better energy management by utilizing different communication modes, and enhanced coexistence using a new channel selection algorithm [8]. Furthermore, BLE version 5.1 is equipped with Angle of Arrival (AoA) and Angle of Departure (AoD) estimation which significantly improves the proximity accuracy of the technology. According to Bluetooth SIG specifications [6], the hardware can support three different types of connections or Physical Layers (PHYs), i.e., BLE 4.x, BLE 5 at $2 \mathrm{Mbps}$, and BLE 5 coded. Here, the PHY BLE 4.X is identified as BLE at $1 \mathrm{Mbps}$ also referred to as LE $1 \mathrm{M}$ PHY. In particular, BLE 5 comes with two new PHYs known as LE $2 \mathrm{M}$ PHY to enhance the throughput, 
and LE Coded PHY to increase the operational range of BLE but with a lower data rate. The impact of multipath fading and internal and external interference is coped with a new channel selection algorithm for choosing the next hopping frequency channel $[9,10]$. These features make BLE 5 a preferred choice for a broad range of IoT applications.

Despite the fact that BLE 5 has been around since 2016, yet it has not been in the limelight of the researchers. The system-level performance evaluation of BLE 5 in an office environment was investigated by Badihi et al. [7]. Böcker et al. [11] studied the suitability of BLE 5 for IoT use cases in terms of scalability, whereas Karvonen et al. [12] provided the performance evaluation of BLE 5 versus BLE 4 . Similarly, Spörk et al. in [13] provided experimental performance analyses of new PHY modes of BLE 5. However, this work emphasizes on the trade-off among different PHY modes and their effects on power consumption and throughput. The same author in another work [14] proposes a mechanism that improves link-layer reliability in BLE while reduces the power consumption. In another work, Pau et al. [4] proposed a fuzzy logic-based solution to manage the power consumption in BLE 5 by varying a transmission power with a fuzzy logic controller while using distance and the battery level as the inputs. To the best of our knowledge, there is no study available in literature related to the use of RSSIbased adaptive PHY selection in BLE 5. In this article, we have proposed an RSSI-based adaptive PHY approach for BLE 5 and evaluated its performance through extensive measurements and simulations in an indoor corridor environment.

The rest of the article is organised as follows. Section 2 provides the overview of BLE 5. Section 3 presents the proposed model for adaptive PHY selection. The description about the measurement setup and simulation methodology is given in Section 4. Section 5 presents a comparison between the measurement and the simulation results and discusses the advantages of the proposed model. Finally, Section 6 concludes the article.

\section{Overview of BLE 5}

BLE is a low-cost, low-energy, and short-range radio technology with high penetration in the market of consumer products. BLE 5 is equipped with new features compared to previous versions which make this technology very attractive for IoT use cases. In this section, we provide an overview of the most important features of BLE 5.

2.1. PHY Modes. BLE 5 is released with the promise of longer range and higher speed compared to previous versions of Bluetooth. These are achieved by introducing three new additional PHY modes from which two of them are encoded. Two coded PHY modes are so-called LE Coded PHY and are used for increasing the communication range, whereas the uncoded LE $2 \mathrm{M}$ PHY is used for high-speed data exchange. It is worth mentioning here that these new PHYs are optional, and only legacy LE $1 \mathrm{M}$ PHY is a mandatory feature. The LE Coded PHY has two versions, i.e., Coded S2 PHY and Coded S8 PHY. The former has Forward Error
Correction (FEC) with 2 coded symbols, and the latter utilizes 8 coded symbols per information. In other words, a single bit of information takes $2 \mu \mathrm{s}$ and $8 \mu$ s resulting in $500 \mathrm{kbps}$ and $125 \mathrm{kbps}$ in Coded S2 PHY and Coded S8 PHY, respectively. All PHYs utilize Gaussian Frequency Shift Keying (GFSK) modulation scheme on the 40 radio channels in the Industrial Scientific and Medical (ISM) band. The LE $2 \mathrm{M}$ PHY is modulated using $2 \mathrm{Msps}$, whereas the rest of the modes are modulated using 1 Msps. In theory, Coded S2 PHY improves the link budget by $5 \mathrm{~dB}$ as compared to $\mathrm{LE}$ $1 \mathrm{M}$ PHY [12].

The structure of the link layer packet for the coded and uncoded PHY modes are shown in Figure 1. As can be seen from the figure, the packet format of the coded and uncoded PHY modes are different. LE $1 \mathrm{M}$ PHY and LE $2 \mathrm{M}$ PHY use the legacy packet format including a preamble, an Access Address, and a Protocol Data Unit (PDU) followed by a CRC. The only difference between the uncoded PHY modes is that LE $2 \mathrm{M}$ PHY uses 2 bytes in the preamble section (refer to Figure 1(a)).

On the other hand, the packet format of coded PHY modes is depicted in Figure 1(b). The packet structure consists of three fields: a Preamble, a FEC Block 1, and a FEC Block 2. The preamble is not coded in order to be able to be received by legacy BLE devices. The FEC Block 1 is composed of an Access Address, a Coding Indicator (CI), and a termination field (TERM 1). This section is always coded by $S=8$ coding regarding the coded PHY type $(S=2$ or $S=$ 8 ). The FEC Block 2 contains a PDU, a CRC, and a TERM 2 fields. This section is either coded by $S=2$ or $S=8$ coding. In order to encode data, the FEC convolutional encoder is firstly used and then spread by the patter mapper. Table 1 tabulates the summary of the existing PHYs and their features in BLE 5.

2.2. Extended and Periodic Advertising. The advertising mode of BLE is an important functionality of the technology which enables devices to transmit data without establishing a connection. This feature enables a myriad of use-cases in IoT such as asset tracking and indoor positioning [15]. During the advertising event, a device that transmits advertising packet on the advertising PHY channels is called advertiser, the device that receives the advertising packet without any intention to connect is called scanner, and the device that is looking for forming an Asynchronous Connection Less (ACL) connection is referred as initiator, which listen for connectable advertising packet. Depending upon the type of the advertisement packet, the scanner or initiator may request to the advertiser which may be followed by a response from the advertiser on the same physical channel [6]. Once the handshake is completed and the connection is established, the initiator becomes the master device and the advertiser becomes the slave device. Bluetooth devices exchange the data packets in a connected state. BLE 5 improves the advertising capability with two new features, namely, extended and periodic advertisement. In previous versions, the advertising data are only transmitted on three advertising channels. BLE 5 offloads more data by using the 37 data channels as well. This is achieved by transmitting a 


\begin{tabular}{|c|c|c|c|}
\hline $\begin{array}{c}\text { Preamble } \\
(1 \text { or } 2 \text { bytes })\end{array}$ & $\begin{array}{c}\text { Access Address } \\
\text { ( } 4 \text { bytes })\end{array}$ & $\begin{array}{c}\text { PDU } \\
\text { ( } 2 \text { to } 257 \text { bytes })\end{array}$ & $\begin{array}{c}\text { CRC } \\
(3 \text { bytes })\end{array}$ \\
\hline
\end{tabular}

(a) The link layer packet format for uncoded LE 1 M PHY and LE 2 M PHY modes

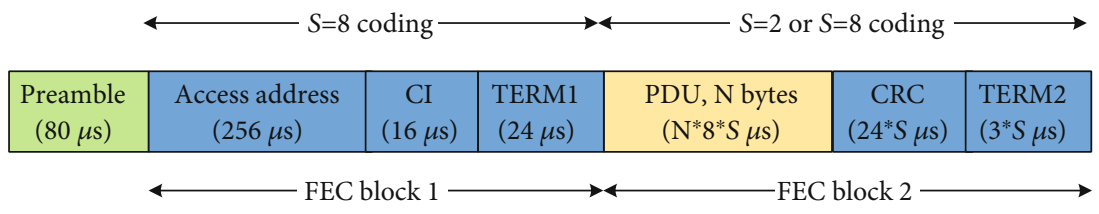

(b) The link layer packet format for Coded S2 PHY and Coded S8 PHY modes

FIgURE 1: The link layer packet formats in BLE 5.

TABLE 1: A summary of PHYs in BLE 5.

\begin{tabular}{lcccc}
\hline \multirow{2}{*}{ PHY } & \multicolumn{2}{c}{ Coding scheme } & \multirow{2}{*}{ Data rate } & \multirow{2}{*}{ Symbol rate } \\
& Header & Payload & & \\
\hline LE 1 M & Uncoded & Uncoded & $1 \mathrm{Mbps}$ & $1 \mathrm{Msps}$ \\
LE 2 M & Uncoded & Uncoded & $2 \mathrm{Mbps}$ & $2 \mathrm{Msps}$ \\
LE coded $S=2$ & $S=8$ & $S=2$ & $500 \mathrm{kbps}$ & $1 \mathrm{Msps}$ \\
LE coded $S=8$ & $S=8$ & $S=8$ & $125 \mathrm{kbps}$ & $1 \mathrm{Msps}$ \\
\hline
\end{tabular}

pointer on three advertising channels, which announces when and on which data channel the data will be transmitted. In this way, the load on three advertising channels reduces which, in turn, improves the functionality of beacons and other connectionless applications.

The periodic advertising is another feature of BLE 5, and it allows the transmission of nonconnected broadcasting data with a fixed interval of time. In this way, both the broadcaster and scanner wake up with a fixed interval of time to exchange data.

2.3. Direction Finding. The Global Positioning System (GPS) is widely used for positioning service in an outdoor environment globally; however, the GPS does not work well in an indoor environment. Therefore, in order to cope with the growing demand for location services in indoors, the feature of direction finding is introduced in BLE 5 [6]. With the help of this feature, the Angle of Arrival (AoA) and the Angle of Departure (AoD) of the Bluetooth signal can be detected, and it helps in tracking the device effectively in three dimensions. The information about the AoA at the receiver side and the AoD at the transmitter side can be used for effective reception and transmission. Other comparative technologies used for the localization are Received Signal Strength Indicator (RSSI), Time of Arrival (ToA), Time of Flight (ToF), and Time Difference of Arrival- (TDoA-) based positioning, whereas AoA and AoD methods belong to the phase-based technology [16]. This direction finding feature has numerous applications, e.g., indoor positioning or navigation, object tracking, and space utilization, and it helps in improving the location prediction precision. In order to use this feature, devices must be equipped with Radio Frequency (RF) switch and an antenna array. Using a simple trigonometry, the angle of arrival or angle of departure can be estimated [16].

\section{Proposed Model}

In the proposed model, it is suggested to use an adaptive approach for selecting the optimal PHY mode of BLE 5 to maximize the throughput. The selection of PHY mode is based on the Received Signal Strength Indicator (RSSI). The information related to the link quality and RSSI is, by default, not directly available to the developers [13]. The challenge is that the BLE specification [6] does not provide any standard way of retrieving the information about the received signal strength or the quality of the link by the master/slave device. An indirect approach for dynamically measuring the link quality is presented in [14]. Similarly, a procedure to acquire the information about the RSSI by master/slave device with a regular interval of time should be crafted.

The flow chart of a proposed adaptive PHY mode selection scheme is shown in Figure 2. During the initialization process, the master and slave device makes a handshake, and after detecting an advertisement from a slave device, the master device initiates a connection request and that is followed by the response from the slave device, and a successful connection is established. After initialization, by default, the LE $1 \mathrm{M}$ PHY mode is selected for data transmission. In order to adapt the physical layer, the RSSI is continuously measured. Now, in order to avoid the ping-pong effect in the PHY mode selection procedure, we have recommended to employ a hysteresis of a few $\mathrm{dBs}$, as it is a common practice to use a hysteresis of a few $\mathrm{dBs}$ in mobility management in cellular networks. We have defined two RSSI threshold levels S1 and S2 for switching to LE 2 M PHY and LE Coded PHY branch from LE $1 \mathrm{M}$ PHY mode, respectively. It is also proposed to use a timer called PHY selection timer to avoid an unnecessary change of PHY mode, caused by any outliers in measured RSSI values. Therefore, in case of meeting the RSSI threshold requirement condition, if the condition of the timer constrained is not passed, then the BLE continues to operate in the same PHY mode as it was operating earlier, and if the condition of the timer constraint is passed, then a new PHY mode is selected for the transmission of data. It is advised to set this timer as greater than or equal to the Connection Interval (CI) time. Now, consider a Bluetooth device operating in LE $1 \mathrm{M}$ PHY mode after the initialization stage as shown in Figure 2; if the measured RSSI + Hys is greater 


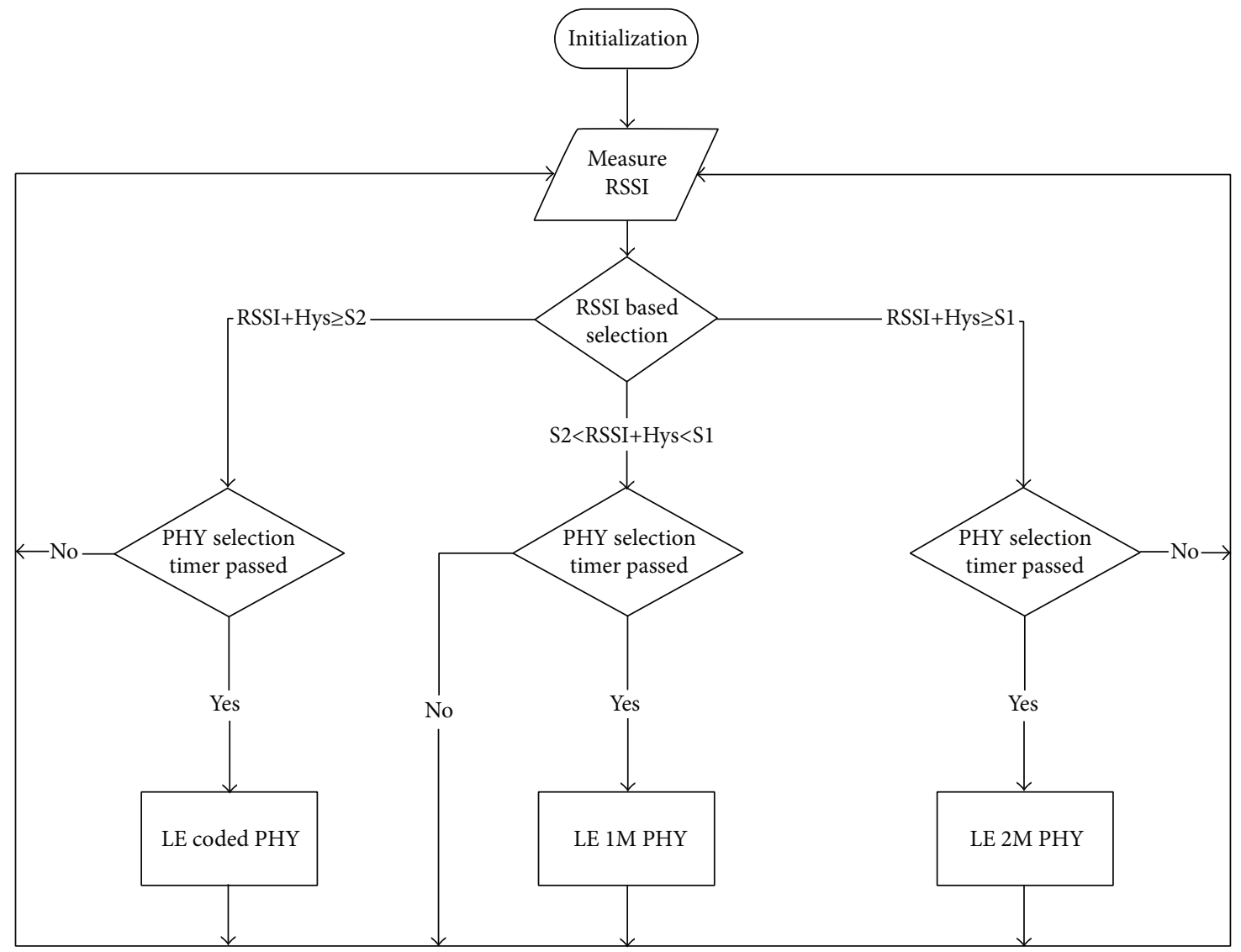

FIgURE 2: Flow chart of adaptive PHY selection for BLE 5.

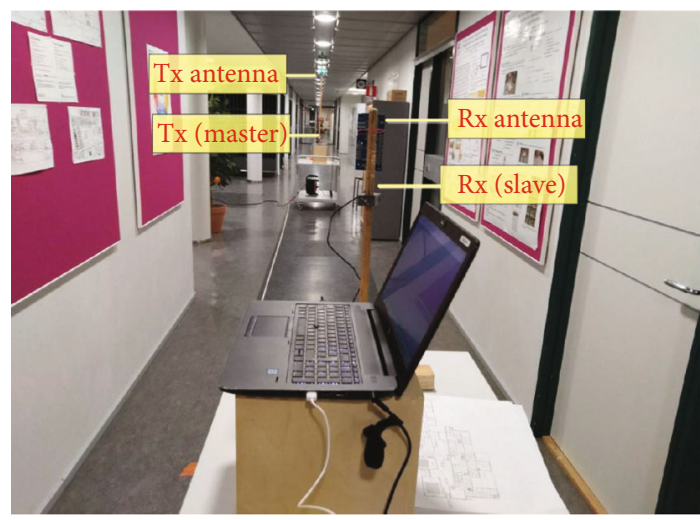

FIGURE 3: Setup for indoor measurements.

than or equals to the S1 threshold and the PHY selection timer condition is fulfilled, then the selected mode will be LE $2 \mathrm{M}$ PHY, and if the PHY selection timer condition is not fulfilled, then the Bluetooth device will continue to operate in earlier selected PHY mode. The Bluetooth device continues to measure RSSI, and at any state, if the measured RSSI + Hys is less than or equals to the S2 threshold and also the PHY selection timer condition is satisfied, then the LE Coded PHY mode will be selected for data transmission. Finally, as long as RSSI + Hys is between S1 and S2, the selected mode of transmission will be LE $1 \mathrm{M}$ PHY.

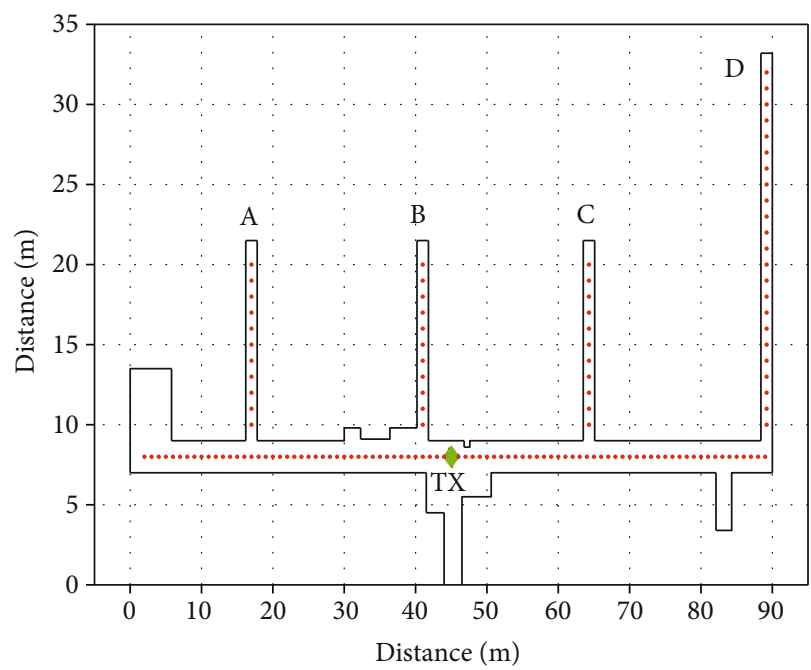

FIGURE 4: Illustration of measurement corridor.

It is important to mention here that according to the BLE 5 specification provided by the Bluetooth SIG in [6], both master and slave can initiate the PHY update procedure, and the master and slave can operate in different PHY modes. From the practical implementation point of view, a Bluetooth device can use the standardized PHY update procedure given by the Bluetooth SIG for BLE 5 in the 
specification [6] to change the selected PHY mode at the runtime. The master or slave can initiate the PHY update procedure. The master may initiate the procedure by sending a $L L_{-}$ $P H Y \_R E Q$ PDU, and the slave responds with $L L_{-} P H Y \_R S P$ PDU. The master confirms the update with $L L_{-} P H Y_{-}$ UPDATE_IND PDU. On the other hand, the slave may also initiate the procedure by sending $L L_{-} P H Y \_R E Q$ PDU, and the master responds with an $L L_{-} P H Y \_U P D A T E \_I N D$ PDU.

\section{Measurement and Simulation Setup}

4.1. Measurement Scenario and Configuration. We have conducted comprehensive measurements in a university corridor environment to evaluate the performance of different PHY modes of BLE 5 from throughput and operational range perspectives. The measurement setup comprises one BLE master device and one BLE slave device. The master device scans for the slave device, whereas the slave device advertises its presence. The master device initiates the BLE connection with the slave after detecting its advertisement. We have used two nRF52840 [17] System on Chips (SoCs) from Nordic semiconductor for this experiment, whereas the nRF52840 is the state-of-the-art SoC module and that integrates multiprotocol $2.4 \mathrm{GHz}$ transceiver with ARM Cortex-M4 processor. The chips are mounted on the masts and connected to the laptops for measurement purposes as depicted in Figure 3. The antenna of the master device is located at 2.5 meters height above the floor to imitate a typical ceilingattached access point deployment. The antenna of the slave is set at 1.5 meters above the ground to mimic a typical height of users' handheld devices. In this experiment, the master device is set at a fixed location in the corridor (green diamond in Figure 4), but the slave is moved in the corridor for each measurement point (red dots in Figure 4). The antenna types for this measurement are omnidirectional. The measurements were conducted on the second floor of the TUAS building at Aalto University. The parameters of the measurements are summarized in Table 2.

4.2. Simulation Setup. One of the targets of this study is to compare and validate the simulation results acquired with the Shoot and Bouncing (SBR) technique $[18,19]$ of the Ray Tracing (RT) with the measurement results. Therefore, initially, the measurement scenario is emulated by using an in-house built 3D RT tool, whereas in the second phase of the simulations, the impact of the adaptive physical layer selection approach is analyzed while utilizing two variants of the transmission power, i.e., $0 \mathrm{dBm}$ and $9 \mathrm{dBm}$. According to the Bluetooth SIG specifications [6], Bluetooth devices of power class 3 have a maximum transmission power of $0 \mathrm{dBm}$, and it is the most commonly used transmission power for Bluetooth devices, whereas BLE 5 devices with power class 1 have a maximum transmission power of $20 \mathrm{dBm}$; however, the nRF52840 chip we have used for measurements has a limit of $9 \mathrm{dBm}$ for maximum transmission power. Therefore, we investigated the impact of $0 \mathrm{dBm}$ and $9 \mathrm{dBm}$ transmission power on the performance of BLE 5 . The three-dimensional floor plan of the corridor of the TUAS building at Aalto University was created in MATLAB
TABLE 2: General measurement parameters.

\begin{tabular}{lc}
\hline Parameter & Value \\
\hline Payload & 27 bytes \\
Connection interval & 7.5 (unit of $1.25 \mathrm{~ms}$ ) \\
Maximum MTU size of ATT & 247 bytes \\
Transmission power & $0 \mathrm{dBm}$ \\
Antenna height of master & $2.5 \mathrm{~m}$ \\
Antenna height of slave & $1.5 \mathrm{~m}$ \\
\hline
\end{tabular}

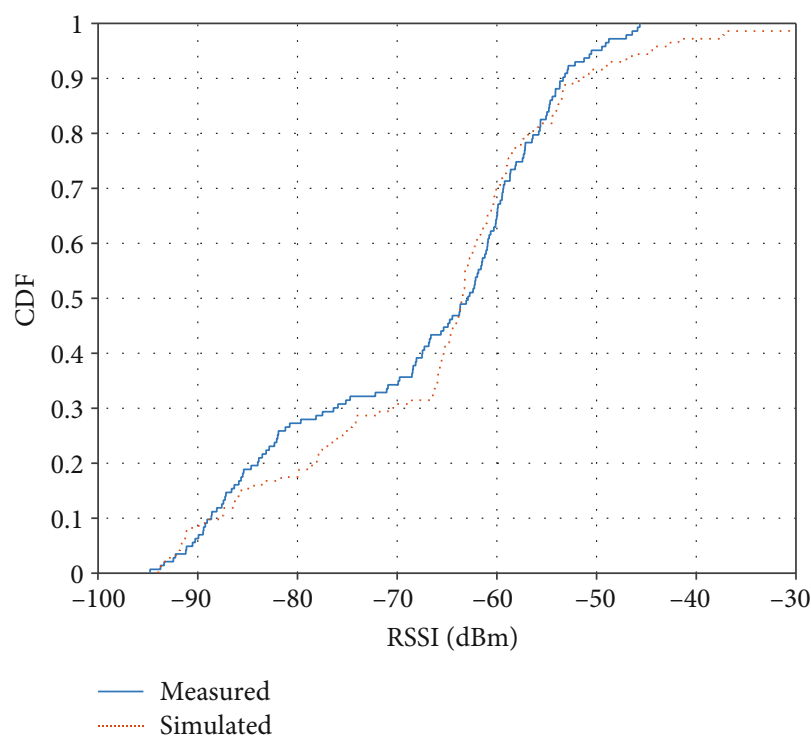

FIGURE 5: CDF of Received Signal Strength Indicator (RSSI).

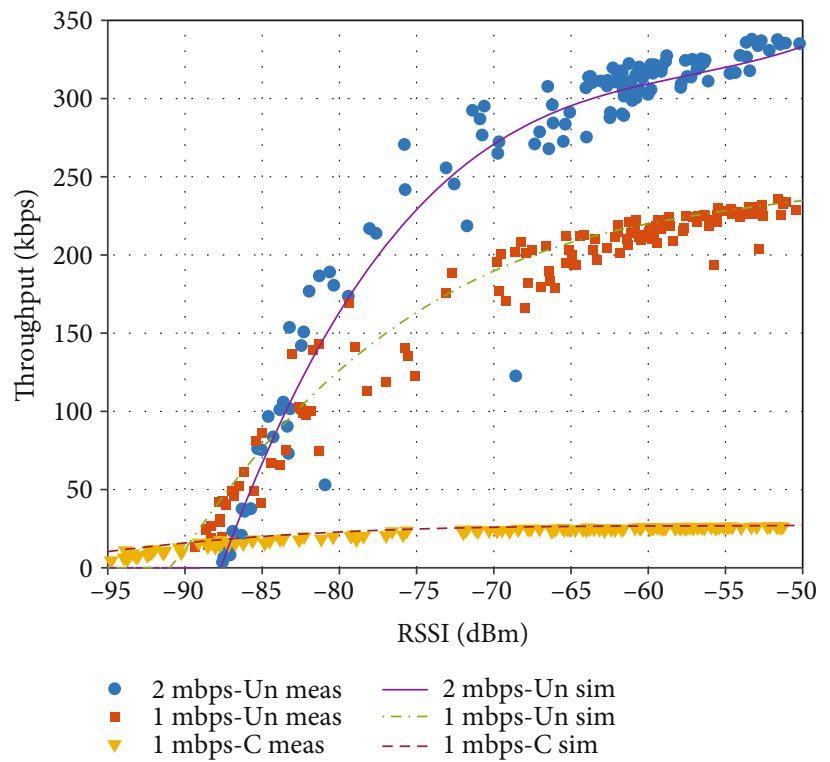

FIgURE 6: Throughput versus RSSI for different physical layers of BLE 5. 
TABLe 3: Root Mean Square Error (RMSE) between the measured values and polynomial equations of different order.

\begin{tabular}{lcccc}
\hline PHYs & RMSE-1st-order polynomial & RMSE-2nd-order polynomial & RMSE-3rd-order polynomial & RMSE-4th-order polynomial \\
\hline 2 Mbps-Un & 40.08 & 24.92 & 22.23 & 21.69 \\
1 Mbps-Un & 23.31 & 13.11 & 12.23 & 12.16 \\
1 Mbps-C & 2.29 & 1.13 & 0.89 & 0.87 \\
\hline
\end{tabular}

for RT simulations; however, only a two-dimensional plan is shown in Figure 4. The measurement points or the position of the slave kit is marked with red dots, whereas the location of the transmitter (TX) also known as the master kit is highlighted by green diamond in Figure 4. In total, 720 rays were launched from the transmitter point with a fixed $0.5^{\circ}$ angular separation between the rays. Each ray continues the propagation until it reaches the maximum number of allowed reflections, i.e., 10 in our simulations. A ray-tracing tool also considers diffracted paths coming from the edges/corners along with the penetrated path through the walls. In the second phase of simulations, in order to acquire a heat map of RSSI over the corridor, the users are homogeneously distributed with $0.5 \mathrm{~m}$ separation.

\section{Results and Discussion}

The first part of this section provides a comparison between the measurements and the simulation results in terms of the RSSI and throughput. Figure 5 presents the Cumulative Distribution Functions (CDFs) of the RSSI for the measured and simulated results and shows a close match between them for the measured received power levels below $-45 \mathrm{dBm}$. It is due to the hardware limitation that we were not able to record the samples above a signal strength of $-45 \mathrm{dBm}$ during the measurement. The Root Mean Square Error (RMSE) between the measured and simulated RSSI was found to be around $5.14 \mathrm{~dB}$. However, when the simulated samples below $-45 \mathrm{dBm}$ were considered only, the RMSE is reduced to $4.6 \mathrm{~dB}$. It highlights that our in-house built ray launching tool adequately models the indoor propagation channel at $2.4 \mathrm{GHz}$ and predicts the received power level with fair accuracy.

Figure 6 shows the scatter plot of the measured throughput against the measured RSSI for three different PHY modes of BLE 5, and it also shows the curves of the throughput acquired by using a proposed RSSI-throughput mapping function. It can be seen in Figure 6 that the measured throughput of different PHY modes is the direct function of the RSSI. Our first target was to propose a simplistic model for the throughput of different PHY modes of BLE 5 as a function of RSSI, and for this purpose, we use the curve fitting toolbox of MATLAB. We tried polynomial equations of different orders and applied a Linear Least Square (LLS) curve fitting method over the measurement data. Table 3 shows the Root Mean Square Error (RMSE) between the measured values and polynomial equations up to the 4 th order. In Table 3, it can be seen that the 2 nd-order polynomial provides large values of the RMSE, whereas the 3rdorder polynomial shows a significant improvement in the RMSE compared to the 2nd-order polynomial, and then,
TABLE 4: Parameters for throughput mapping function.

\begin{tabular}{lccccccc}
\hline PHYs & $A$ & $B$ & $C$ & $D$ & $E$ & $x_{1}$ & $x_{2}$ \\
\hline 2 Mbps-Un & $8.97 \mathrm{e}^{-3}$ & 1.54 & 90.15 & 2112 & 340 & -87 & -50 \\
1 Mbps-Un & $2.98 \mathrm{e}^{-3}$ & 0.46 & 24.9 & 704 & 235 & -90 & -50 \\
1 Mbps-C & $0.3 \mathrm{e}^{-3}$ & 0.052 & 3.03 & 86.1 & 27 & -95 & -50 \\
\hline
\end{tabular}

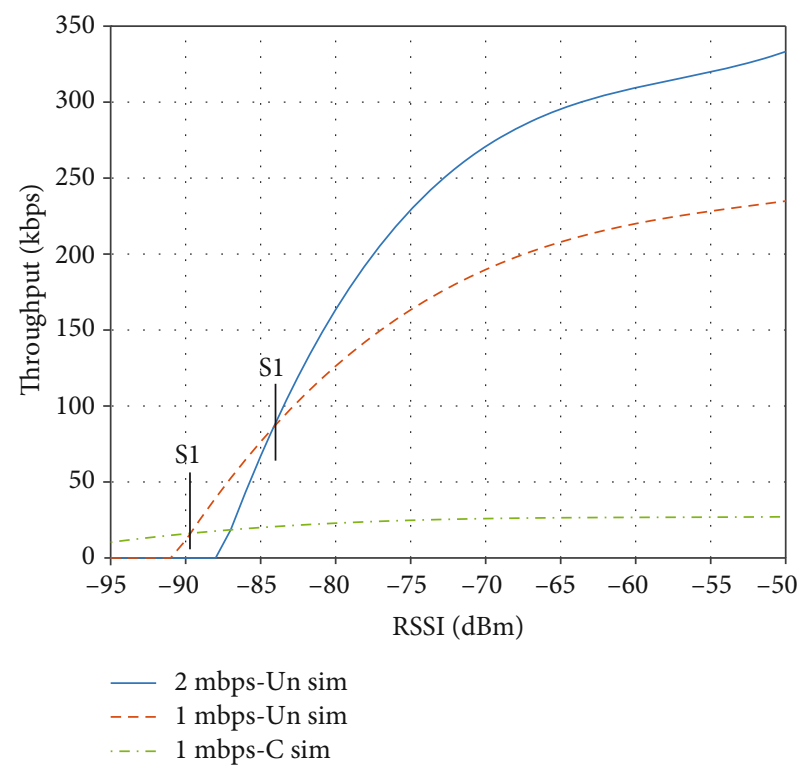

FIGURE 7: Graph for adaptive physical layer selection of BLE 5.

the improvement in the RMSE starts to get saturated with higher-order polynomial, i.e., 4th-order polynomial. The problem of overfitting also arises with the 4th-order polynomial, and the values of the coefficients become very small. Therefore, we have selected a 3rd-order polynomial for the RSSI-throughput mapping function, as it provides a good compromise between the complexity of the model and the accuracy of the acquired results.

The generalized form of our proposed RSSI-based throughput mapping function for different PHY modes of BLE 5 as a third-order polynomial is shown in eq. (1), where $T$ is the throughput in kbps, $P_{r}$ is the RSSI in $\mathrm{dBm} ; A, B, C$, and $D$ are the coefficients; and $E$ is the maximum supported data rate in kbps. We have used extensive measurement data and the curve-fitting tool to derive the values of these coefficients for different PHYs of BLE 5, and they are presented in Table 4. It is critical to mention that eq. 1 and the values of coefficients of eq. (1) for different PHY modes presented in Table 4 are valid for any scenario, as in eq. (1) the throughput is directly mapped over the RSSI, whereas the RSSI to 
throughput mapping is independent of the environment. For our proposed adaptive PHY selection method, the RSSIbased throughput mapping function is a piece-wise function and is a combination of RSSI-throughput mapping function of all three PHYs.

$$
T\left(P_{r}\right)=\left(\begin{array}{lll}
A P_{r}^{3}+B P_{r}^{2}+C P_{r}+D, & \text { if } & x_{1} \leq P_{r} \leq x_{2}, \\
E, & \text { if } & P_{r}>x_{2}, \\
0, & \text { if } & P_{r}<x_{1} .
\end{array}\right.
$$

In Figure 6, it can be seen that the samples of throughput against the RSSI are spread over a larger range for LE $2 \mathrm{M}$ PHY as compared to other PHY modes. In Figure 6, it can be observed from the measurement data that in the region with the RSSI above $-80 \mathrm{dBm}$, the LE $2 \mathrm{M}$ PHY layer clearly provides a higher data rate compared with other considered physical layers, and it is definitely a preferred choice for the transmission. The measurement data shows that a maximum data rate of $340 \mathrm{kbps}$ was achieved with LE $2 \mathrm{M} \mathrm{PHY,} \mathrm{whereas}$ the maximum data rate of LE $1 \mathrm{M}$ PHY and LE Coded PHY were limited to $235 \mathrm{kbps}$ and $27 \mathrm{kbps}$, respectively. It must be noted that LE $2 \mathrm{M}$ PHY offers almost $45 \%$ more peak data rate relative to LE $1 \mathrm{M}$ PHY while utilizing $100 \%$ extra bandwidth. It is also important to highlight that LE $2 \mathrm{M}$ PHY has the shortest coverage and the data connection is lost at the RSSI below $-86 \mathrm{dBm}$. Similarly, the data connection is terminated for LE $1 \mathrm{M}$ PHY and LE Coded PHY at the RSSI below $-90 \mathrm{dBm}$ and $-95 \mathrm{dBm}$, respectively. However, the data rate provided by the LE Coded PHY scheme is quite low compared with the other two physical layers, but the communication range is extended with the coded scheme. It is given in [12] that theoretically, Coded S2 PHY improves the link budget by $5 \mathrm{~dB}$ as compared to LE $1 \mathrm{M}$ PHY, whereas we have utilized Coded S8 PHY in our measurement campaign, and from the acquired measurement results, it can be deduced and validated that the LE Coded PHY provides an extended range of nearly $8 \mathrm{~dB}$ and $5 \mathrm{~dB}$ compared with LE $2 \mathrm{M}$ PHY and LE $1 \mathrm{M}$ PHY, respectively.

Figure 7 shows the graphs of the throughput acquired through the mapping function for different PHY modes. The target is to select the PHY mode with a maximum data rate. On the basis of the measurement data and the RSSIthroughput mapping function, Figure 7 highlights two switching points, i.e., S1 $(-84 \mathrm{dBm})$ and S2 $(-90 \mathrm{dBm})$ for switching to LE $2 \mathrm{M}$ PHY and LE Coded PHY from the LE $1 \mathrm{M}$ PHY mode, respectively. Later, we have used these thresholds for evaluating the performance of our proposed RSSI-based adaptive PHY selection approach.

In the second part of this article, the whole corridor of the TUAS building in Aalto University is considered for the simulations, and a heat map of the RSSI is acquired through the ray launching simulations with $0 \mathrm{dBm}$ transmission power as shown in Figure 8. For better understanding and visualization of the data, the CDFs of the RSSI for two different transmission power are presented in Figure 9. Due to transmission power restrictions for $\mathrm{BLE}$ at $2.4 \mathrm{GHz}$ in some areas, the

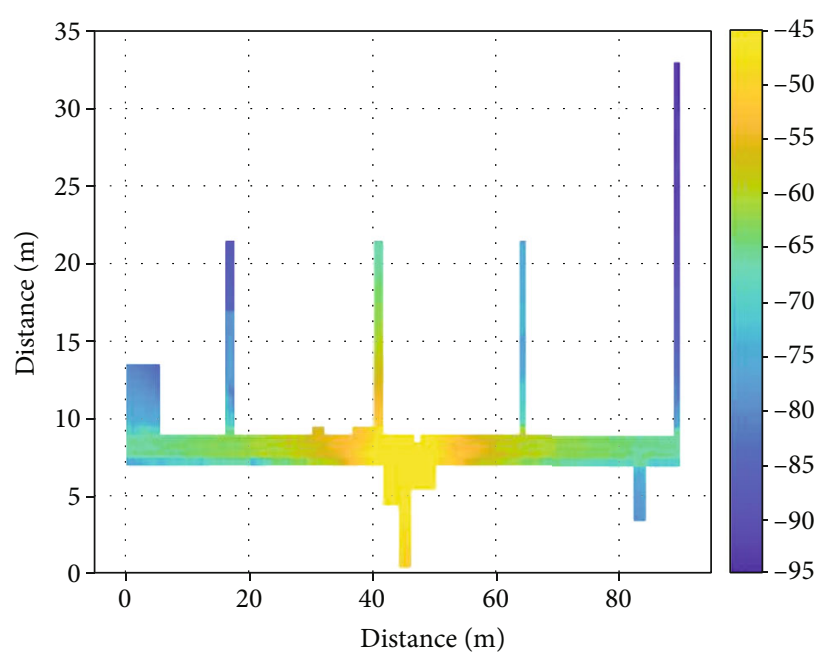

Figure 8: Heat map of the received signal strength indicator.

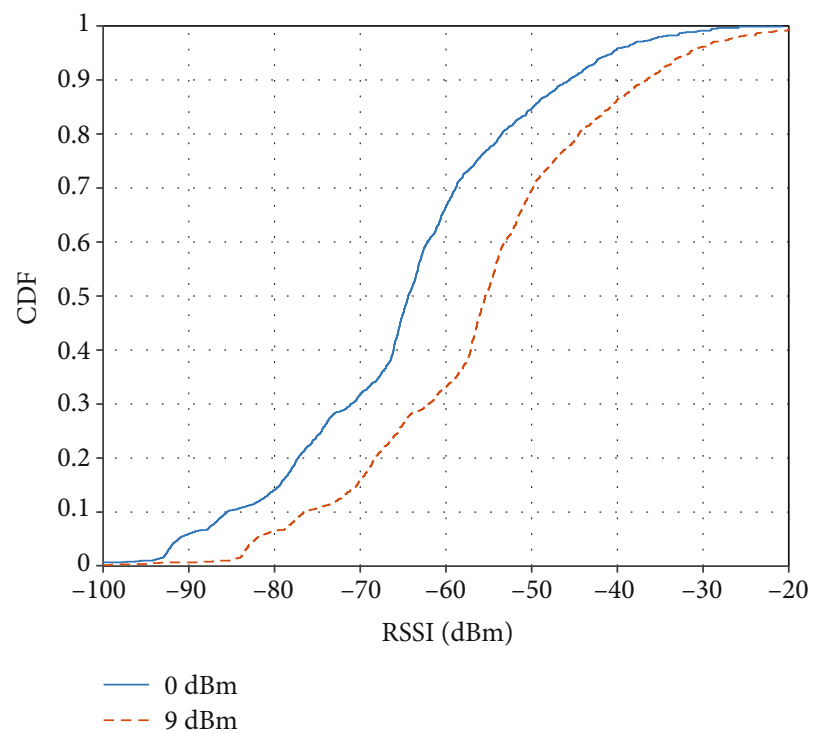

FIGURE 9: CDF of RSSI in the office corridor with different transmission power.

transmission with $9 \mathrm{dBm}$ is not always viable. It can be deduced from the results shown in Figures 8 and 9 that almost the whole corridor except the deep space in wing $D$ has RSSI better than $-95 \mathrm{dBm}$ with a single transmitter in the middle of the corridor with $0 \mathrm{dBm}$ transmission power. The mean RSSI level of $-64.8 \mathrm{dBm}$ is obtained with $0 \mathrm{dBm}$ transmission power. The CDF curve of the RSSI is simply right-shifted by increasing the transmission power by $9 \mathrm{~dB}$ in Figure 9. However, it will be interesting to see the impact of the increase in transmission power on the throughput and outage ratio of the system.

Figure 10(a) and Figure 10(b) show the CDFs of the throughput acquired with different physical layers with $0 \mathrm{dBm}$ and $9 \mathrm{dBm}$ transmission power, respectively, and Figure 11 shows the bar graphs of the outage ratio. It is critical to analyze both these performance indicators together for better understanding. The simulated throughput is obtained 


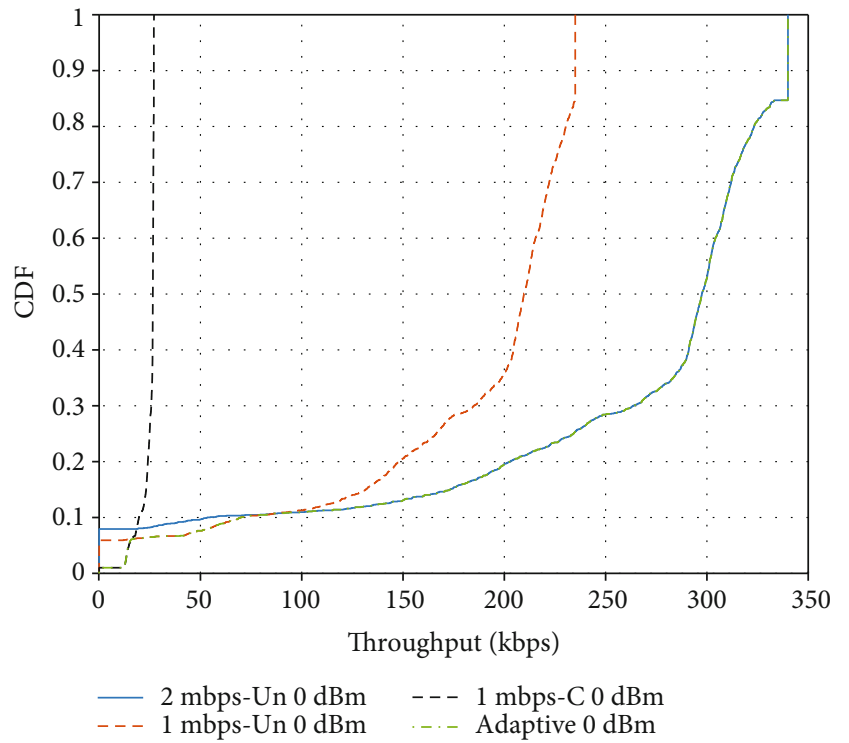

(a)

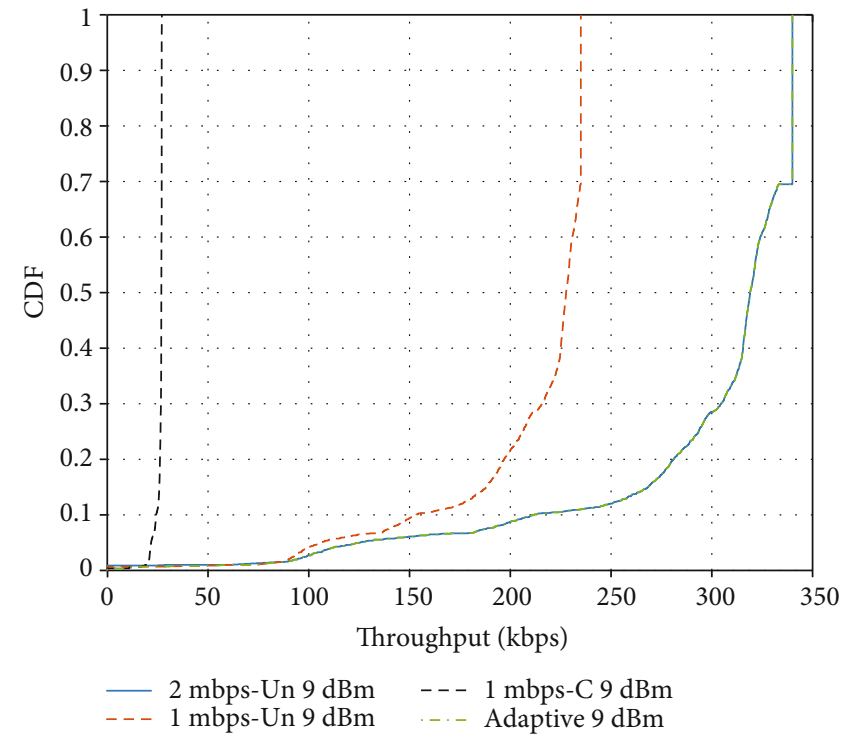

(b)

Figure 10: CDF plot of throughput for different physical layers of BLE 5 with (a) $0 \mathrm{dBm}$ and (b) $9 \mathrm{dBm}$ transmission power.

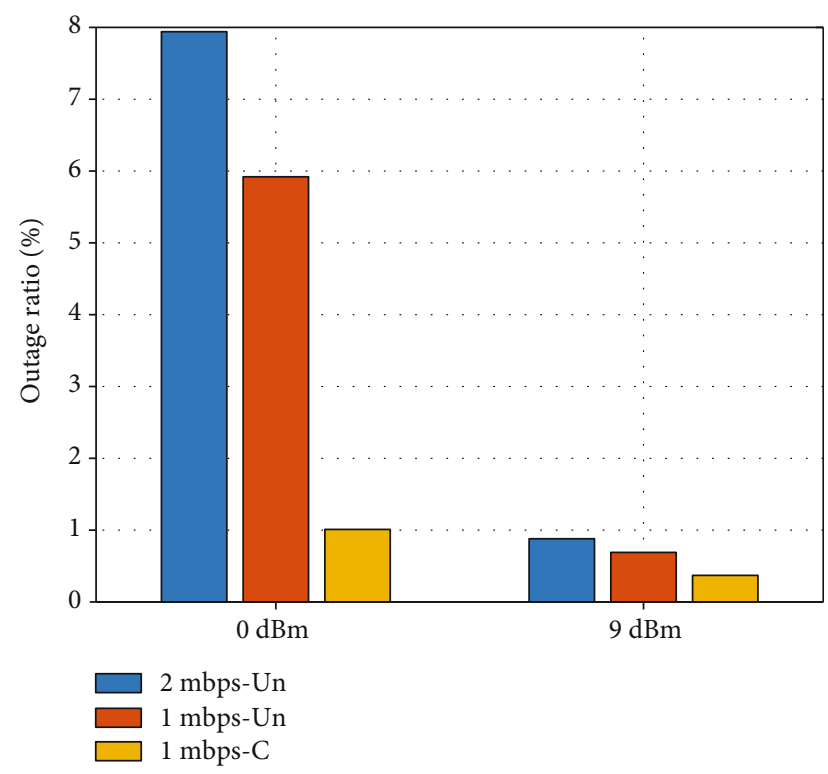

FIGURE 11: Bar plot of outage ratio for different physical layers of BLE 5.

by using the RSSI-throughput mapping function given in eq. (1). It can be seen in Figure 10 that the receiver points with the high RSSI, i.e., above $-50 \mathrm{dBm}$, are limited by the maximum supported data rate of each physical layer. In the case of $1 \mathrm{Mbps}$ coded PHY with $0 \mathrm{dBm}$ transmission power, around $70 \%$ of the samples are with the data rate between 25.7 and $27 \mathrm{kbps}$, and offer a mean throughput of $24.9 \mathrm{kbps}$ as shown in Table 4. These numbers show the inefficiency of the coded PHY in the area with a good or nominal received signal strength. However, the outage ratio is nearly $1 \%$ with the coded PHY. On the other hand, with the $0 \mathrm{dBm}$ transmission power, the $2 \mathrm{Mbps}$ and $1 \mathrm{Mbps}$ uncoded PHYs offer a mean throughput of $257.7 \mathrm{kbps}$ and $184.2 \mathrm{kbps}$, with $7.94 \%$ and $5.92 \%$ outage ratio, respectively. It is important to highlight here that in Figure 9, the graphs of the adaptive PHY approach mainly overlap over the results of $2 \mathrm{Mbps}$ uncoded; therefore, the results of $2 \mathrm{Mbps}$ uncoded PHY is not clearly visible. While considering the static position of master and slave devices, it was found that by utilizing the proposed adaptive PHY selection scheme, the overall throughput is slightly improved compared to the 2 Mbps uncoded PHY, and the mean throughput of $259.7 \mathrm{kbps}$ is achieved with $1 \%$ outage ratio as shown in Table 5. It shows that the proposed PHY selection method not only improves the mean throughput of the system but also enhances the outage ratio.

Generally, in wireless/cellular networks, the five percentile (5\%tile) and ten percentile (10\%tile) samples are used to represent the cell edge users, and those users or locations are considered in critical condition. Therefore, we have also analyzed the median, 5\%tile, and 10\%tile of the throughput along with the mean value. Interestingly, the 5\%tile of the throughput with $0 \mathrm{dBm}$ transmission power is zero for both LE $1 \mathrm{M}$ PHY and LE $2 \mathrm{M}$ PHY modes as the outage ratio is above 5 in both cases, whereas the LE Coded PHY and our proposed adaptive PHY mode selection approach offer a $5 \%$ tile throughput of $14.7 \mathrm{kbps}$. It is interesting to note in Table 5 that with $0 \mathrm{dBm}$ transmission power, the $10 \%$ tile throughput of $69.8 \mathrm{kbps}$ achieved with LE $1 \mathrm{M} \mathrm{PHY} \mathrm{is} \mathrm{higher}$ than the $10 \%$ tile throughput of $53.7 \mathrm{kbps}$ obtained with LE $2 \mathrm{M}$ PHY. The $10 \%$ tile throughput with LE Coded PHY is $19.6 \mathrm{kbps}$ which is just $5.3 \mathrm{kbps}$ less than the mean throughput of the LE Coded PHY mode.

Similarly, with the $9 \mathrm{dBm}$ transmission power, the overall RSSI level is improved which in turn boosts the throughput and outage ratio of the system. The mean throughput of the system with $9 \mathrm{dBm}$ transmission power is $298.4,211$, and $26.1 \mathrm{kbps}$ with $0.88 \%, 0.69 \%$, and $0.37 \%$ outage ratio for $2 \mathrm{Mbps}$ uncoded, $1 \mathrm{Mbps}$ uncoded, and $1 \mathrm{Mbps}$ coded PHY, respectively, whereas the adaptive PHY selection offers a 
TABLE 5: Throughput and outage ratio of different PHY modes.

\begin{tabular}{|c|c|c|c|c|c|c|}
\hline & $\begin{array}{l}\text { Transmit power } \\
(\mathrm{dBm})\end{array}$ & $\begin{array}{c}\text { Mean throughput } \\
\text { (kbps) }\end{array}$ & $\begin{array}{l}\text { Median throughput } \\
\text { (kbps) }\end{array}$ & $\begin{array}{l}5 \% \text { tile throughput } \\
\text { (kbps) }\end{array}$ & $\begin{array}{l}10 \% \text { tile throughput } \\
(\mathrm{kbps})\end{array}$ & $\begin{array}{c}\text { Outage ratio } \\
(\%)\end{array}$ \\
\hline 2 Mbps-Un & 0 & 257.7 & 297.5 & 0 & 53.7 & 7.94 \\
\hline $1 \mathrm{Mbps}-\mathrm{Un}$ & 0 & 184.2 & 209.7 & 0 & 69.8 & 5.92 \\
\hline $1 \mathrm{Mbps}-\mathrm{C}$ & 0 & 24.9 & 26.5 & 14.7 & 19.6 & 1.01 \\
\hline Adaptive & 0 & 259.7 & 297.5 & 14.7 & 69.8 & 1.01 \\
\hline 2 Mbps-Un & 9 & 298.4 & 319.0 & 125.2 & 211.2 & 0.88 \\
\hline $1 \mathrm{Mbps}-\mathrm{Un}$ & 9 & 211.2 & 227.7 & 106.1 & 152.9 & 0.69 \\
\hline $1 \mathrm{Mbps}-\mathrm{C}$ & 9 & 26.1 & 26.8 & 21.8 & 24.3 & 0.37 \\
\hline Adaptive & 9 & 298.6 & 319.0 & 125.2 & 211.2 & 0.37 \\
\hline
\end{tabular}

TABLE 6: PHY mode usage distribution using adaptive PHY mode selection.

\begin{tabular}{lcc}
\hline & $\begin{array}{c}0 \mathrm{dBm} \text { transmit power } \\
(\%)\end{array}$ & $\begin{array}{c}9 \mathrm{dBm} \text { transmit power } \\
(\%)\end{array}$ \\
\hline 2 Mbps-Un & 89.23 & 98.42 \\
1 Mbps-Un & 4.85 & 0.88 \\
1 Mbps-C & 5.92 & 0.69 \\
\hline
\end{tabular}

mean throughput of $298.6 \mathrm{kbps}$ with $0.37 \%$ outage ratio. It was found that the use of higher transmission power, i.e., $9 \mathrm{dBm}$ compared to $0 \mathrm{dBm}$, has a significant impact on the $5 \%$ tile and $10 \%$ tile throughput samples of LE $1 \mathrm{M} \mathrm{PHY} \mathrm{and}$ LE $2 \mathrm{M}$ PHY communication mode. It can be seen in Table 5 that the 5\%tile and 10\%tile throughput values of LE $1 \mathrm{M}$ PHY and LE $2 \mathrm{M}$ PHY have improved by a healthy margin. The results presented in Table 5 show that the adaptive PHY selection approach has high gain and is more beneficial in the case of regions with mixed RSSI samples, i.e., with a mix of low and high RSSI values. It is important to mention here that in our simulations, we have only considered the corridor area of the office building; however, the advantage of the adaptive PHY mode selection approach will be more evident in the case where the Bluetooth coverage in office rooms is also considered.

Table 6 shows the utilization of three different BLE 5 communication modes while employing the proposed adaptive PHY mode selection procedure in our simulation considering static master and slave positions with $0 \mathrm{dBm}$ and $9 \mathrm{dBm}$ transmission power. It was found that with $0 \mathrm{dBm}$ transmission power, $89.23 \%$ of the corridor area was covered with LE $2 \mathrm{M}$ PHY, and only $4.85 \%$ and $5.92 \%$ of the area were supported by LE $1 \mathrm{M}$ PHY and LE Coded PHY, respectively. Altogether, it makes $99 \%$, whereas nearly $1 \%$ of the area had coverage outage. It shows that most of the time, LE $2 \mathrm{M}$ PHY is the preferred choice for the adaptive PHY selection approach, and the contribution from other modes is small. The usage of LE $2 \mathrm{M}$ PHY was further increased to $98.42 \%$ by increasing the transmission power to $9 \mathrm{dBm}$.

It is critical to mention here that we have considered static measurement points for measurements and for the ray launching simulations, whereas in the future, our target is to study the impact of mobility and also investigate the impact of the hysteresis and PHY selection timer on the performance of the proposed adaptive physical layer selection algorithm in BLE 5.

\section{Conclusions}

This article presents a simple and easy-to-implement RSSIbased adaptive PHY selection approach for selecting an optimal physical layer for the connection mode of BLE 5. An RSSI-throughput mapping function for different PHYs of BLE 5 is provided in this article, and this algorithm also defines the threshold levels for switching between the PHYs. The communication range and throughput of different PHYs of BLE 5 in a typical large office corridor environment are evaluated and validated by measurements and 3D RT simulations. A small RMSE error of about $4.6 \mathrm{~dB}$ was found between the measured and the simulated RSSI. The measurement results show that the data connection is terminated at RSSI levels below $-87 \mathrm{dBm},-90 \mathrm{dBm}$, and $-95 \mathrm{dBm}$ for $\mathrm{LE}$ $2 \mathrm{M}$ PHY, LE $1 \mathrm{M}$ PHY, and LE Coded PHY, respectively, and that shows the range of the extended coverage with coded schemes. However, the simulation results reveal that the adaptive PHY selection approach provides two-way benefits as it enhances the mean throughput of the system while maintaining a low outage ratio. It is also shown that by using a higher transmission power, the overall throughput and outage ratio can be improved.

\section{Data Availability}

The data in the form of results is included in the manuscript. The rest, if needed, the data can be made available on request by sending a request to the corresponding author of this paper at the email address muhammad.sheikh@aalto.fi.

\section{Conflicts of Interest}

The authors declare that they have no conflicts of interest.

\section{Acknowledgments}

This work was partly supported by the Finnish public funding agency for research, Business Finland under the project code 6388/31/2018 5G-FORCE. 


\section{References}

[1] A. Al-Fuqaha, M. Guizani, M. Mohammadi, M. Aledhari, and M. Ayyash, "Internet of things: a survey on enabling technologies, protocols, and applications," IEEE Communications Surveys \& Tutorials, vol. 17, no. 4, pp. 2347-2376, 2015.

[2] E. Sisinni, A. Saifullah, S. Han, U. Jennehag, and M. Gidlund, "Industrial internet of things: challenges, opportunities, and directions," IEEE Transactions on Industrial Informatics, vol. 14, no. 11, pp. 4724-4734, 2018.

[3] J. Lin, W. Yu, N. Zhang, X. Yang, H. Zhang, and W. Zhao, “A survey on internet of things: architecture, enabling technologies, security and privacy, and applications," IEEE Internet of Things Journal, vol. 4, no. 5, pp. 1125-1142, 2017.

[4] G. Pau, M. Collotta, and V. Maniscalco, "Bluetooth 5 energy management through a fuzzy-PSO solution for mobile devices of internet of things," Energies, vol. 10, no. 7, p. 992, 2017.

[5] W. Bronzi, R. Frank, G. Castignani, and T. Engel, "Bluetooth low energy performance and robustness analysis for intervehicular communications," Ad Hoc Networks, vol. 37, pp. 76-86, 2016.

[6] "Bluetooth Core Specification, The Bluetooth Special Interest Group," https://www.bluetooth.com/specifications/bluetoothcore-specification.

[7] B. Badihi, F. Ghavimi, and R. Jäntti, "On the system-level performance evaluation of Bluetooth 5 in IoT: Open office case study," in 2019 16th International Symposium on Wireless Communication Systems (ISWCS), pp. 485-489, Oulu, Finland, 2019.

[8] K.-H. Chang, "Bluetooth: a viable solution for IoT? [Industry perspectives]," IEEE Wireless Communications, vol. 21, no. 6, pp. 6-7, 2014.

[9] T. Watteyne, S. Lanzisera, A. Mehta, and K. S. J. Pister, "Mitigating multipath fading through channel hopping in wireless sensor networks," in 2010 IEEE International Conference on Communications, pp. 1-5, Cape Town, South Africa, 2010.

[10] N. Baccour, A. Koubâa, L. Mottola et al., "Radio link quality estimation in wireless sensor networks," ACM Transactions on Sensor Networks, vol. 8, no. 4, pp. 1-33, 2012.

[11] S. Böcker, C. Arendt, and C. Wietfeld, "On the suitability of Bluetooth 5 for the internet of things: performance and scalability analysis," in 2017 IEEE 28th Annual International Symposium on Personal, Indoor, and Mobile Radio Communications (PIMRC), pp. 1-7, Montreal, QC, Canada, 2017.

[12] H. Karvonen, C. Pomalaza-Ráez, K. Mikhaylov, M. Hämäläinen, and J. Iinatti, "Experimental performance evaluation of ble 4 versus ble 5 in indoors and outdoors scenarios," in Advances in Body Area Networks I, pp. 235-251, Springer, 2019.

[13] M. Spörk, C. A. Boano, and K. Römer, "Performance and trade-offs of the new PHY modes of BLE 5," in Proceedings of the ACM MobiHoc Workshop on Pervasive Systems in the IoT Era - PERSIST-IoT '19, pp. 7-12, Catania, Italy, July 2019.

[14] M. Spörk, J. Classen, C. A. Boano, M. Hollick, and K. Römer, "Improving the reliability of bluetooth low energy connections," EWSN, pp. 144-155, Junction Publishing, USA, 2020.

[15] B. Badihi, J. Zhao, S. Zhuang, O. Seppänen, and R. Jäntti, "Intelligent construction site: on low cost automated indoor localization using bluetooth low energy beacons," in 2019 IEEE Conference on Wireless Sensors (ICWiSe), pp. 29-35, Penang Island, Malaysia, 2019.
[16] S. Lehtimaki, Understanding Advanced Bluetooth Angle Estimation Techniques for Real-Time Locationing, Silicon Labs, Tech. Rep, 2018, https://www.silabs.com/documents/public/ presentations/ew-2018-understanding-advanced-bluetoothangle-estimation-techniques-for-real-time-locationing.pdf.

[17] “nRF52840," https://www.nordicsemi.com/Products/Lowpower-short-range-wireless/nRF52840.

[18] M. U. Sheikh, F. Ghavimi, K. Ruttik, and R. Jäntti, “Analysis of indoor solutions for provision of indoor coverage at $3.5 \mathrm{GHz}$ and $28 \mathrm{GHz}$ for 5G system," in 2019 26th International Conference on Telecommunications (ICT), pp. 340-345, Hanoi, Vietnam, 2019.

[19] R. A. Kipp and M. C. Miller, "Shooting-and-bouncing ray method for 3D indoor wireless propagation in WLAN applications," in IEEE Antennas and Propagation Society Symposium, 2004, pp. 1639-1642, Monterey, CA, USA, 2004. 